Article

\title{
Modeling and Recipe Optimization of Anti-Glare Process Using Sandblasting for Electronic Display Glass
}

\author{
Chul Hong Min ${ }^{1}$, Yoon Sung Kang ${ }^{1}$ and Tae Seon Kim ${ }^{2, *}$ (D) \\ 1 Central Research Institute, SYNOPEX Inc., Hwasung 18423, Korea; ves1110@naver.com (C.H.M.); \\ kys82@synopex.com (Y.S.K.) \\ 2 School of Information, Communications and Electronics Engineering, Catholic University of Korea, \\ Bucheon 14662, Korea \\ * Correspondence: tkim@catholic.ac.kr; Tel.: +82-2-2164-4367
}

Received: 6 November 2020; Accepted: 28 November 2020; Published: 2 December 2020

\begin{abstract}
Recently, anti-glare (AG) surface treatment technology has been considered as a standard process to enhance the visibility of electronic display devices. For AG, the hydrofluoric acid (HF)-based chemical etch method is the most common approach for the current display glass industry. However, in order to overcome the environmental and durability degradation problems of the HF-based chemical etch method, this paper proposes an eco-friendly physical surface treatment technology using the sandblasting method. Based on the preliminary analysis results using the central composite design (CCD) method-based response surface modeling methodology (RSM), additional experiments and analyses were performed for process modeling and optimal process recipe generation. To characterize the sandblasting process, the mean value of haze was considered as the process output, and the pressure of the nozzle, the distance of the nozzle from the surface of glass, the glass feed rate, and the grit size of the abrasives were considered as process inputs. Based on the process model using the statistical response surface regression method and machine learning-based approaches, the proposed method can generate optimized process recipes for various haze targets of $10 \%, 20 \%$, and $30 \%$, with an average haze difference of $0.84 \%, 0.02 \%$, and $0.86 \%$, and maximum deviations of $1.26 \%$, $1.14 \%$, and $1.4 \%$, respectively. Through the successful completion of this work, it is expected that the proposed surface treatment method can be applied to various products including mobile phones, tablet PCs, and windshields of vehicles.
\end{abstract}

Keywords: sandblasting; anti-glare (AG); haze; electronic display glass; process modeling; central composite design (CCD) method-based response surface modeling methodology (RSM)

\section{Introduction}

To evaluate the performance of conventional consumer electronic display devices including television (TV), cathode ray tube (CRT), or liquid crystal display (LCD) computer monitors, the resolution, refresh rate, brightness, contrast ratio, and chromaticity of the display were considered as common key assessment items. However, as the usage of mobile devices increases, outdoor visibility, readability, and visual fatigue are also considered very important factors for quality measures [1]. Conventional evaluation factors are primarily related to the characteristics of the display devices, but the visibility, readability, and visual fatigue in lighting or bright outdoors are highly related to the light reflection phenomenon of glass used on the surface of the display devices [2]. For this reason, in the display used for medical diagnosis, testing criteria related to reflection were set and quality assurance (QA) evaluation of the display device was performed [3]. In general, there are two approaches to solve 
the light reflection problem in electronic displays, the anti-reflective (AR) surface treatment method and the anti-glare (AG) surface treatment method. AR surface treatment technology negates reflected light by coating a film layer with a different refractive index on a glass substrate [4]. AG surface treatment technology typically uses light diffusion by roughening a reflective surface or coating small particles on the surface [5]. In the AR method, surface reflection is minimized by the formation of small porous or nano-patterns that can lower the index of film refraction or by applying a single or multi-layer of AR coating [6]. Owing to this capability, AR technology is very useful in various applications that require precise control of light, such as solar cell fabrication processes and semiconductor manufacturing processes, as well as electronic displays [7]. However, since AR surface treatment methods require multiple steps of the coating process or precise nano-patterning process, they require high processing costs for mass production of electronic display glass [8]. AG surface treatment has a problem that the scattering layer for roughening the surface degrades the display's clarity [5], but it is widely used for mass production since it can alleviate the specular reflection problem at relatively low manufacturing costs through direct etching of the glass surface [9]. Typically, in the electronic display industries, for low-cost processing, the hydrofluoric acid (HF) etching solution-based chemical etching is widely used for surface treatment of AG. However, the chemical etching technique has the disadvantage of lowering the durability of the glass due to the chemical reactions between the hydrofluoric acid and the composition materials in the glass. In addition, HF is a very hazardous chemical with very strong worldwide regulations. Therefore, the development of alternative technology for surface treatment of AG is essential to overcome the environmental regulations and safety problems. To this end, in this paper, we present an AG surface treatment method that can satisfy various haze target requirements using the sandblasting process. Sandblasting or abrasive-blasting is a physical-mechanical technique using fine particle type abrasives which are accelerated through high-speed air or gas through a nozzle and then etched through a physical impact on a hard surface. Classically, sandblasting has been mostly used in the cleaning process to remove paint or rust. However, recently sandblasting has been used to improve material properties in various fields. To enhance the film adhesion in the material coating process, sandblasting was used for the formation of rough surface or formation of micro patterns on the surface areas [10]. Sandblasting can be applied to the solar cell fabrication process to improve power generation efficiency by applying a surface treatment to minimize light reflection [11], and sandblasting has been applied to make super-hydrophobic surface characteristics [12]. Sandblasting is also used to form about $50-\mu \mathrm{m}$ microstructures at a relatively low production cost by using an abrasive with a smaller particle size (around $30 \mu \mathrm{m}$ ) compared to the conventional method. In this case, it is sometimes called powder blasting or micro-powder blasting to distinguish it from conventional sandblasting [13]. Sandblasting is generally used for glass to make a double glass structure by roughening the surface of the glass, or to make a pattern or frosting effect on the glass surface for aesthetic purposes [14]. In this case, the sandblasting is generally a simple process that is not significantly affected by process variation. Recently, research of surface treatment methods that can be applied to mass production of electronic display glass using sandblasting has begun to replace the existing HF-based chemical process [15]. There has been a growing demand in the electronics industry for AG glass with specific haze value for functional and aesthetic advantages, not just at the level of simple pattern formation using AG. For example, many high-end level mobile phones released after 2019 are manufactured to have surface characteristics that are strong against fingerprints and aesthetics while maintaining durability for the backside cover glass as well as the AG treatment for display cover glass for visibility. For these applications, different from the conventional sandblasting process, it is essential to develop a stringent surface treatment process that minimizes variation with a target haze value. For this, in this paper, we proposed an eco-friendly physical surface treatment technology using a sandblasting method for various haze targets. As described above, we can see that sandblasting was used to form a pattern on the glass surface or to use it for surface treatment to reduce the reflectivity, but the relevant research on the process model between various haze values and the sandblasting process is not confirmed other than the previous research results of this research team [15]. Based on our preliminary analysis 
results using the design of experiment (DOE) and statistical analysis, additional experiments and analysis were performed for process modeling and optimal process recipe generation for various haze targets. To characterize the sandblasting process, the mean value of haze was considered as the process output, and the pressure of the nozzle, the distance of the nozzle from the surface of glass, the motor speed for glass feed speed control, and the particle size of the abrasives were considered as process inputs. To evaluate mass production feasibility, mean of haze, uniformity of haze, glossiness, surface roughness, and transmittance were measured and analyzed for final samples.

The structure of this paper is as follows. In Section 2, three experimental methods are described for modeling, model evaluation, and mass production testing that satisfies various haze targets. In Section 3, the results of process modeling using response surface regression and machine learning are compared, and the feasibility of mass production of the process recipe determined using the prediction of the process model is described and finished with the conclusion.

In this work, three sets of experiments were conducted for process modeling, model validation test, and evaluation of optimized recipes for the feasibility of mass production. Through the response surface regression-based statistical model and machine learning-based process model, the optimal process recipes were defined to satisfy the various haze targets, and the final test for the evaluation of mass production feasibility showed acceptable performance results for the haze targets of $10 \%, 20 \%$, and $30 \%$.

\section{Materials and Methods}

Figure 1 shows the overall flow of the proposed method for modeling and recipe generation of the sandblasting process for AG surface treatment. In this study, three sets of experiments were conducted for process modeling, the model validation test, and evaluation of optimized recipes for the feasibility of mass production. First, 54 experiments were conducted for modeling of the sandblasting process. Based on the first experiment set, two models, a statistical model and a machine learning-based model, were designed and the second experiment set was used for the validation of the modeling performance. In the second experiment, the experiments were conducted by reducing the range of the process variables compared to the first experiment set, and 30 experiments were used for the evaluation of the modeling performance. Based on modeling validation test results, optimal process recipes were defined, and final experiments were conducted to evaluate whether the defined process recipes could satisfy the various haze targets, and whether haze uniformity, glossiness, and transmittance values were acceptable for mass production.

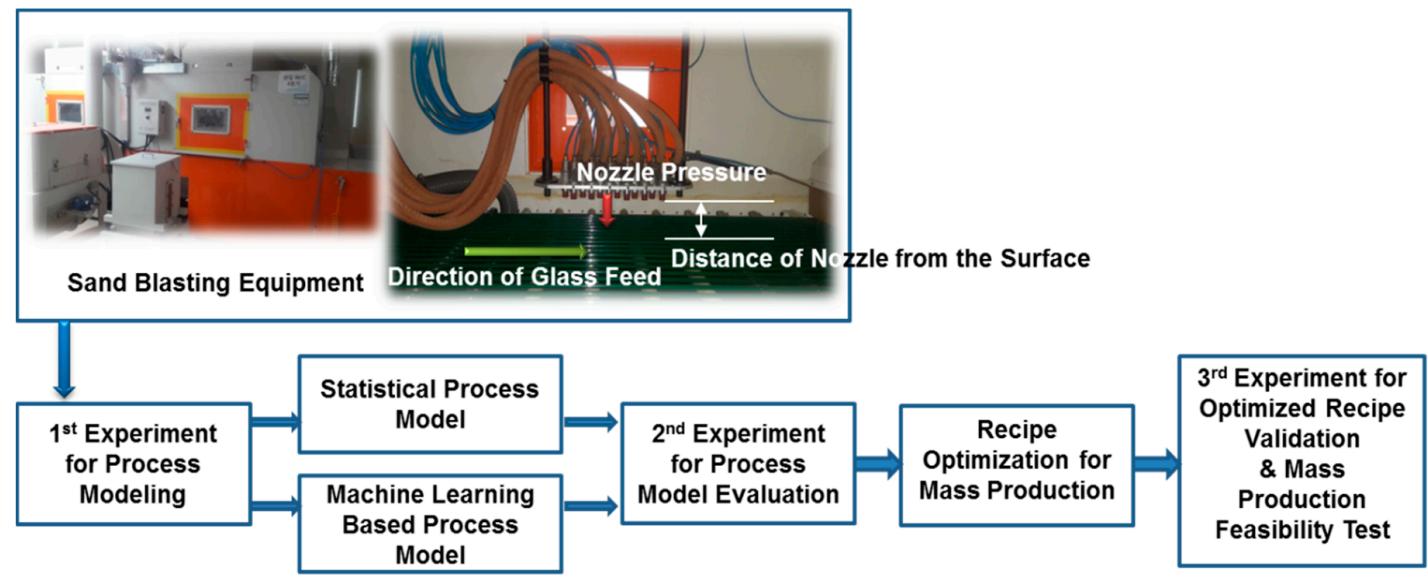

Figure 1. The flow of the proposed method for modeling and recipe generation of the sandblasting process for anti-glare (AG) surface treatment. 


\subsection{Experimental Design for Process Modeling}

In this paper, the range of process variables was determined using our preliminary statistical analysis results [15] and the number of outputs for the modeling of process characteristics was adjusted. In the previous preliminary work of this research team, the pressure of the nozzle, the distance of the nozzle from the surface of glass, and the glass feed rate were used as process input variables for sandblasting, and roughness, haze, surface durability, and flatness were used as process outputs for the evaluation of mechanical process characteristics [15]. For surface roughness expression, arithmetic average roughness $(\mathrm{Ra})$ was calculated with measured values using a laser microscope [16]. Haze characteristics were quantized by measuring the amount of scattered or reflected light while passing through the AG surface-treated glass using measurement equipment from Minolta, and the surface durability values were measured by applying a force to the AG-treated glass to measure the magnitude of the force at the time when the glass substrate was fractured. For the flatness, the degree of warpage was expressed by using the difference between the maximum and minimum values after measuring five places of the specimen using a laser bending measurement device. In the preliminary experiment, a total of 20 experiments were conducted on three input factors using the face-centered central composite design (CCD) method with 2 blocks. Through the preliminary analysis, it was confirmed that the surface durability and flatness did not show statistical significance on the input ranges of the process variables considered in this study. Therefore, in this study, the surface durability and flatness were excluded from the process outputs for process modeling. Table 1 shows the process inputs and their ranges targeted in this study based on the results of preliminary works. In this work, in order to understand the effects of three inputs (the pressure of the nozzle, the distance of the nozzle from the surface, and the motor speed for control of glass feed speed) on the sandblasting process, we tested separately for each abrasive with two types of grit size (\#600 and \#800) as shown in Table 1. Among the three process input variables for each model with different grit sizes, one input variable was varied according to the minimum step size shown in Table 1, and the other two input variables were fixed at the median value of the variation range; that is, 27 experiments were conducted for each abrasive type, and a total of 54 experiments were performed.

Table 1. Process inputs and their ranges.

\begin{tabular}{cccc}
\hline Parameter & Range (or Type) & Units & $\begin{array}{c}\text { Input Resolution } \\
\text { (Minimum Step Size to Control) }\end{array}$ \\
\hline $\begin{array}{c}\text { Pressure of the nozzle } \\
\text { Distance of the nozzle from the surface }\end{array}$ & $2.5 \sim 4.5$ & $\mathrm{MPa}$ & 0.25 \\
Motor speed for control of glass & $200 \sim 280$ & $\mathrm{~mm}$ & 10 \\
feed speed & $8 \sim 12$ & $\mathrm{RPM}$ & 0.5 \\
Particle (or grit) size of the abrasives & $\# 600, \# 800$ & $\mathrm{~N} / \mathrm{A}$ & $\mathrm{N} / \mathrm{A}$ \\
\hline
\end{tabular}

For sandblasting abrasives, the grit size number (\#600, \#800, etc.) was based on the agreed-upon particle size distribution for each mesh or screen size [17]. In other words, the average particle size of abrasive \#600 was from $13 \mu \mathrm{m}$ to $16 \mu \mathrm{m}$, and abrasive \#800 was from 9.8 to $12.3 \mu \mathrm{m}$. Usually, when the grit size is smaller than \#600, it is called micro-fine grit, and if the process is classified by the size of the grit, the sandblasting process we used in this work might be considered to belong to "micro-powder blasting". The glass substrate used in this work was a mobile phone display glass manufactured by Corning co. (Gorilla 2320) with a size of $50 \times 90 \mathrm{~mm}$ and thickness of $0.3 \mathrm{~mm}$ to $0.5 \mathrm{~mm}$.

\subsection{Experiments for Testing of Developed Process Models}

The second experiment was used to evaluate the modeling performance of two developed models (statistical process model and machine learning-based process model) using the data from the first experiments. Since the purpose of the second experiment was not to support data for process modeling but for the evaluation of modeling performance, the process condition of the second experiment was defined based on the engineer's experience rather than a systemic experimental design; that is, 
at first, through the analysis of the first experiment result, the most dominant input factor $\left(\mathrm{p}_{1}\right)$ for the sandblasting process was found. Next, the variation width of the process output was checked while changing the values of the $\mathrm{p}_{1}$. In this case, the remaining process variables were fixed to a specific value according to the first experiment result and the decision of the engineer as shown in Table 2.

Table 2. The process ranges for testing of the developed process model.

\begin{tabular}{cccc}
\hline Haze Target (\%) & Abrasive Type & $\begin{array}{c}\text { Constant Inputs } \\
10\end{array}$ & Range of Pressure (MPa) \\
\hline 20 & $\# 600$ & $\begin{array}{c}\text { Distance }=240 \mathrm{~mm}, \\
\text { speed }=10 \mathrm{RPM} \\
\text { Distance }=250 \mathrm{~mm}, \\
\text { speed }=10 \mathrm{RPM} \\
\text { Distance }=230 \mathrm{~mm}, \\
\text { speed }=10 \mathrm{RPM}\end{array}$ & $2 \sim 3$ \\
30 & $\# 600$ & $\begin{array}{c}\text { Distance }=250 \mathrm{~mm}, \\
\text { speed }=10 \mathrm{RPM} \\
\text { Distance }=230 \mathrm{~mm}, \\
\text { speed }=10 \mathrm{RPM} \\
\text { Distance }=220 \mathrm{~mm}, \\
\text { speed }=10 \mathrm{RPM}\end{array}$ & $3 \sim 4$ \\
30 & $\# 800$ & & $2.25 \sim 3.25$ \\
\hline
\end{tabular}

\subsection{Final Experiment for Validation of Process Recipes}

After the process recipes for each haze target of $10 \%, 20 \%$, and $30 \%$ were determined based on the modeling result, the final experiment was carried out using five glass substrates for each haze target using the defined recipes. To review the mass production feasibility, five glass substrates were processed simultaneously on the same panel and the haze was measured in three places of each glass; that is, to see the deviation of surface treatment, the uniformity was calculated using the measured fifteen haze values for each haze target. In addition, glossiness, roughness, and transmittance were also measured to see the feasibility of mass production.

\section{Results and Discussion}

\subsection{Response Surface Regression-Based Statistical Model}

Table 3 shows the estimated regression coefficients of $\# 800$ grit size based on the response surface regression method. In this regression, the R-squared value was 0.9781 . As shown in this table, the pressure of the nozzle and the distance of the nozzle from the surface of glass were most dominant to the haze. This could be inferred that the increase in pressure may lead to an increase in the glass etch rate, and thus it affects the increase of haze. On the contrary, it was found that the higher the value of the distance of the nozzle from the surface of glass, the lower the etch rate, so it affects the decrease of haze value. In addition, in this model, the motor speed for control of glass feed speed could not show the statistical significance on the basis of a $p$-value of 0.05 . However, the square term of motor speed for control of glass feed speed was shown to have statistical significance based on a $p$-value of 0.05 ; that is, the effect of the motor speed for control of glass feed speed was not significant within the corresponding process range, but it was inferred that the effect was amplified by the square of this input parameter and had an effect toward reducing the haze value.

Table 4 shows the estimated regression coefficients of \#600 grit size based on the response surface regression method. In this regression, the R-squared value was 0.9895 . As a result of comparing the coefficients of the sandblasting model with \#600 grit size with coefficients of the sandblasting model with \#800 grit size, the number of each coefficient value was different, but most of the modeling input process parameters including the pressure of the nozzle, the distance of the nozzle from the surface of glass, and the squared term of the motor speed for control of glass feed speed had the same tendency on haze. 
Table 3. The estimated regression coefficients for the sandblasting model using \#800 abrasive.

\begin{tabular}{cccc}
\hline Term & Coefficient & $\boldsymbol{t}$-Value & $\boldsymbol{p}$-Value \\
\hline Constant & 17.3218 & 68.915 & 0.000 \\
Pressure & 8.7573 & 19.687 & 0.000 \\
Distance & -9.6742 & -21.748 & 0.000 \\
Speed & 0.3231 & 0.726 & 0.476 \\
Pressure $\times$ Pressure & 0.6991 & 1.127 & 0.273 \\
Distance $\times$ Distance & 2.1107 & 3.404 & 0.003 \\
Speed $\times$ Speed & -2.0208 & -3.259 & 0.004 \\
\hline
\end{tabular}

Table 4. The estimated regression coefficients for the sandblasting model using \#600 abrasive.

\begin{tabular}{cccc}
\hline Term & Coefficient & $t$-Value & $p$-Value \\
\hline Constant & 25.978 & 67.694 & 0.000 \\
Pressure & 20.243 & 29.805 & 0.000 \\
Distance & -19.696 & -28.999 & 0.000 \\
Speed & -4.154 & -6.116 & 0.000 \\
Pressure $\times$ Pressure & 3.964 & 4.187 & 0.000 \\
Distance $\times$ Distance & 8.425 & 8.899 & 0.000 \\
Speed $\times$ Speed & -1.781 & -1.881 & 0.075 \\
\hline
\end{tabular}

In the coefficient comparison of two models, the motor speed for the control of glass feed speed was the only input variable with a different sign. However, we could ignore this mismatch since this parameter showed the statistical insignificance for the sandblasting model of \#800 grit size on the basis of a $p$-value of 0.05 . However, the model of $\# 600$ grit size showed statistical significance on the motor speed for control of the glass feed speed and it could be inferred that the etch rate of \#600 material was higher than that of \#800 abrasive material because the grit size of \#600 was larger than that of \#800; thus, only the sandblasting model of \#600 grit size showed that haze was related on motor speed for the control of the glass feed speed. However, as in the case of the sandblasting model of \#800 grit size, the effect of the motor speed for the control of the glass feed speed did not seem to be large compared to other process inputs.

Figure 2 is the response surface regression modeling result of the sandblasting process of the \#600 grit size. This plot shows the effects of the pressure of the nozzle and the distance of the nozzle from the surface of glass on haze. At this plot, the speed was fixed as the center point of the processing range that was used in the experiment. As shown in the 3D surface plot in Figure 2a, the pressure of the nozzle and the distance of the nozzle from the surface of glass were critical factors for the haze change. In this plot, the haze value was relatively high at the higher pressure and lower at the distance of the nozzle. Especially when the distance of the nozzle from the surface of glass was less than $225 \mathrm{~mm}$, the increase rate of the haze became more rapid. These effects are better illustrated in the contour plot in Figure 2b. For example, where the distance of the nozzle from the surface of glass was $250 \mathrm{~mm}$, the haze varied from $0 \%$ to $45 \%$ depending on the pressure change. On the other hand, if the distance was around $200 \mathrm{~mm}$, the variation in the haze according to the change in nozzle pressure exceeded $75 \%$.

Figure 3 shows the effects of the motor speed for control of glass feed speed and the distance of the nozzle from the surface on the haze using the response surface regression modeling result of the sandblasting process of \#600 grit size. At this plot, the nozzle pressure was fixed as the center point of the processing range that was used in the experiment. As shown in Figure 3a, compared to the distance of the nozzle from the surface of glass, the effect of the motor speed for control of glass feed speed on the haze was relatively insignificant. But the haze value tended to decrease as the motor speed for the control of the glass feed speed increased at the same distance of the nozzle from the surface of the glass. All the lines of the contour plot in Figure $3 \mathrm{~b}$ are drawn in the vertical direction, and as described in Figure 3a, this implies that the effect on the haze of the motor speed for control of glass feed speed was insignificant relative to the distance of the nozzle from the surface of the glass. In the 
plot of Figure 3b, the vertical direction lines tilt to the left along with the increase of the motor speed for the control of the glass feed speed, which is assumed to be because the glass cannot be sufficiently etched to achieve high haze values under high-speed conditions.

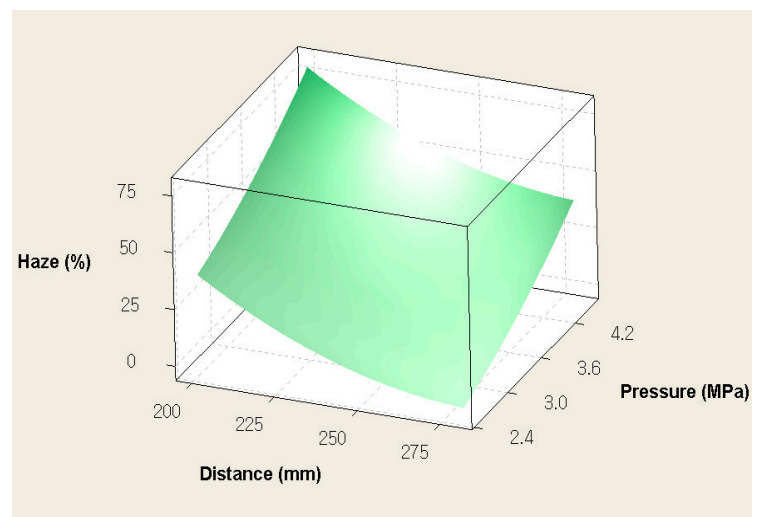

(a)

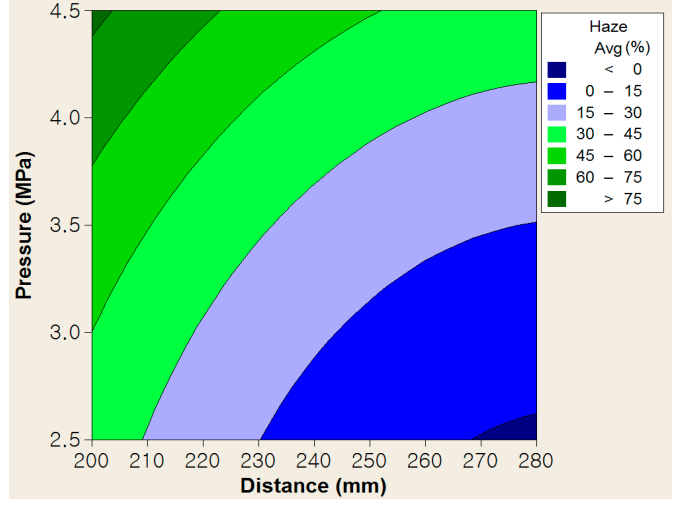

(b)

Figure 2. Effects of the distance of the nozzle from the surface and the pressure of the nozzle on the haze (motor speed was hold at 10 RMP): (a) 3D surface plot; (b) contour plot.

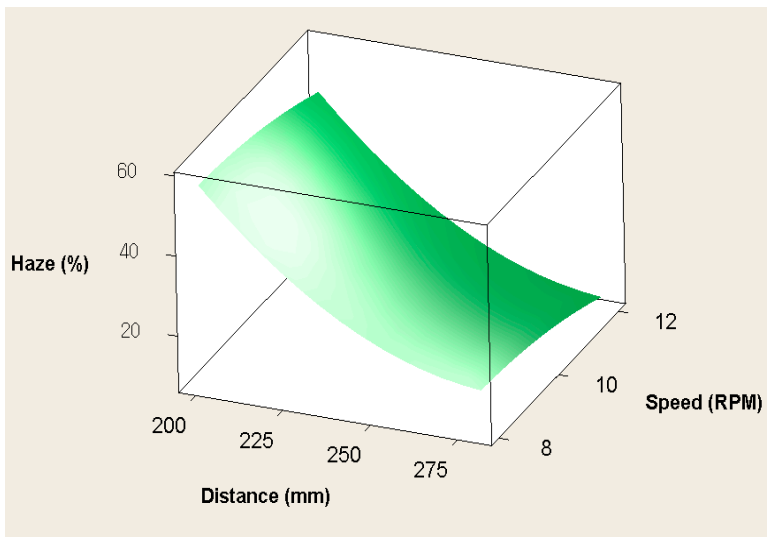

(a)

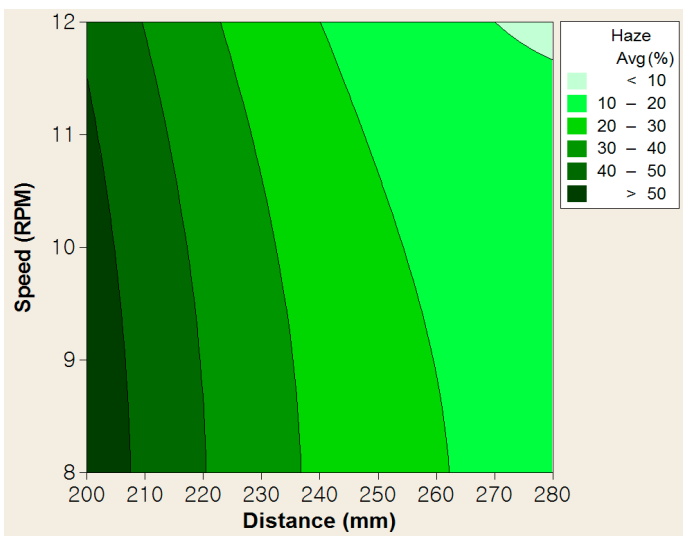

(b)

Figure 3. Effects of the distance of the nozzle from the surface and the motor speed for control of glass feed speed on the haze (the pressure of nozzle was held at 3.5 Mpa: (a) 3D surface plot; (b) contour plot.

\subsection{Machine Learning-Based Process Model}

In this paper, a modified design of the experiment was used to add more detailed experiments to preliminary research using CCD experimental design. For modification of the experimental design, an expert engineer of the sandblasting process set the two process input values to constant and changed only one input variable within process ranges. With this set of experiments, a statistical regression model was developed using the second-degree polynomial-based response surface regression model. However, in this set of experiments, extra experiments that do not follow standard DOE were added to use prior knowledge based on the experience of sandblasting experts. In addition, considering that the accuracy of modeling may be limited due to the order limitation of the second-degree polynomial model, machine learning was additionally implemented in process modeling. Machine learning has been steadily applied and studied in various fields to model high-order complex processes. Typically, compared to statistical regression models, the machine learning-based modeling approaches require a relatively big size of training data. However, there are research results that show superior modeling results compared to statistical methods even if only limited experimental results are used due to the critical time and cost limitations for experimental design such as semiconductor processes [18]. Therefore, in this paper, the sandblasting process was modeled using the WEKA (Waikato Environment 
for Knowledge Analysis) machine learning platform with limited training data [19]. To maximize modeling performance, learning algorithms and their hyper-parameters were selected and optimized by the Auto-WEKA package [20]. Similar to the statistical process models, for machine learning-based models, each sandblasting process was modeled according to its grit size. Based on the Auto-WEKA package, the Gaussian process classifier was selected for modeling of the sandblasting process with the \#600 grit size, and the locally weighted learning (LWL) method was selected for modeling of the sandblasting process with the \#800 grit size. The Gaussian process classifier used in this package use Gaussian process for regression without hyper-parameter tuning [21]. The Gaussian process is a kind of random stochastic process and is defined by a collection of random variables with a multivariable Gaussian distribution. This Gaussian distribution is specified by its mean function and covariance function which characterizes the correlation between different points in the function [22]. Here, the Gaussian process regression calculated the probability distribution of suitable functions using training data. To make a prediction using the Gaussian process, we needed the covariance kernel function, and the normalized polykernel, polykernel, and radial basis function (RBF) kernel were applied in this WEKA package [23]. The LWL method used in this package uses an instance-based algorithm to assign instance weight and use naïve Bayes classification [24]. In the WEKA package, the naive Bayes model plays the role of linear regression and the locally weighted learning method was used to train the data with the lazy training approach. Through the LWL method, the attribute independence issue of naïve Bayes classifier can be solved [25].

The data used for modeling is the same as the statistical model. In this case, the amount of data is very limited for machine learning, so all 27 experimental data were used for training. In other words, we could not divide this experimental data into a training set and validation set at this time. Instead, additional experiments for model evaluation were conducted later. Table 5 shows the modeling results of the machine learning-based process model. As a result of machine learning-based process modeling, the relative absolute errors of the $\# 600$ grit size model and the $\# 800$ grit size model were $7.05 \%$ and $7.44 \%$, respectively, showing no significant difference.

Table 5. The modeling error of machine learning-based process models.

\begin{tabular}{cccc}
\hline Process to Model & Correlation Coefficient & Relative Absolute Error & Root Relative Squared Error \\
\hline$\# 600$ grit size & 0.9973 & $7.05 \%$ & $7.38 \%$ \\
$\# 800$ grit size & 0.9966 & $7.44 \%$ & $8.43 \%$ \\
\hline
\end{tabular}

Figure 4 shows the effects of the pressure of the nozzle and the distance of the nozzle from the surface of the glass on haze using the Gaussian process classifier-based modeling result of the sandblasting process of \#600 grit size. At this plot, the speed was fixed as the center point of the processing range that was used in the experiment as shown in the previous analysis of the statistical model. As shown in the 3D surface plot in Figure 4a, the tendency of haze to increase as the nozzle pressure increased and the distance of the nozzle from the surface of the glass decreased was consistent with the statistical response surface methodology (RSM) modeling result. However, when the distance and pressure values were close to the minimum and maximum values of the processing range considered in this work, the haze values were close to $100 \%$, indicating an unusual modeling prediction. This is presumed to be an over-generalization error of the process model when there was insufficient data for training. Comparing the contour plot in Figure $4 b$ with that of Figure $3 b$, the trend of these two plots was similar, but it appears that Figure $4 \mathrm{~b}$ shows a higher non-linearity on process inputs and represents an excessive haze value at the minimum or maximum of the process input conditions. 


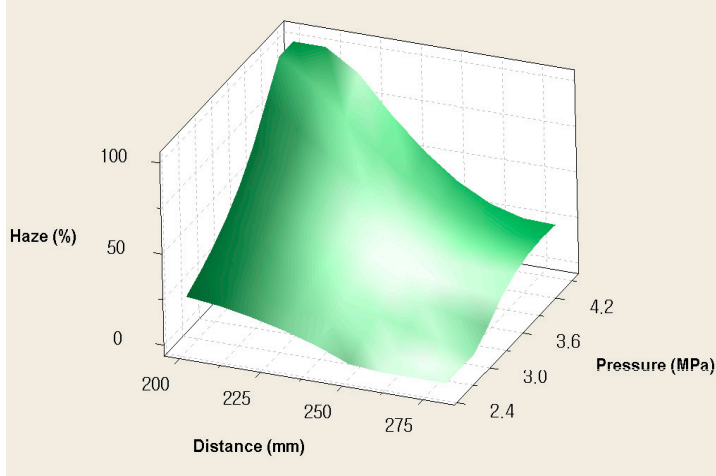

(a)

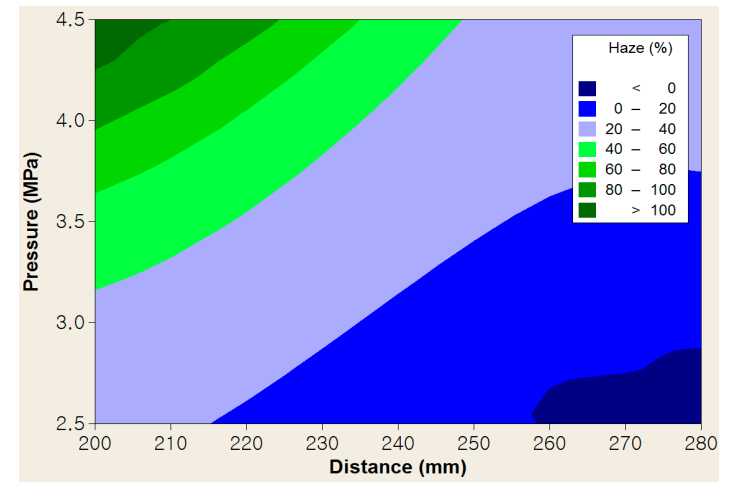

(b)

Figure 4. Effects of the distance of the nozzle from the surface of the glass and the nozzle pressure on the haze: (a) 3D surface plot; (b) contour plot.

Figure 5 shows the effects of the motor speed for control of glass feed speed and the distance of the nozzle from the surface on haze using the Gaussian process classifier-based modeling result of the sandblasting process of \#600 grit size. At this plot, the nozzle pressure was fixed as the center point of the processing range as we did in the previous analysis. As shown in this plot, the effect of the motor speed for control of glass feed speed and the pressure of nozzle on haze was much different from that of the statistical process model. At a relatively high distance of the nozzle from the surface of the glass, the haze was shown to decrease with increasing speed, which is generally consistent with the results of the statistical process model. However, at low-distance values, the haze values tended to increase as the increase of motor speed for control of glass feed speed. As shown in Figure 5, when the distance of the nozzle from the surface of glass and the motor speed for control of glass feed speed approached the minimum and maximum values of the processing range, respectively, the haze value increased rapidly to reach $100 \%$. These prediction results do not match with the practical processing results and these wrong modeling results were due to the lack of training data. The contour plot in Figure $5 \mathrm{~b}$ shows that the tendency of haze to increase as the distance value decreased was consistent with the statistical model, but the model of the contour plot suddenly changed around 250 to 260 in the distance range of the nozzle from the surface of the glass and it is presumed to have occurred due to the overfitting or generalization error.

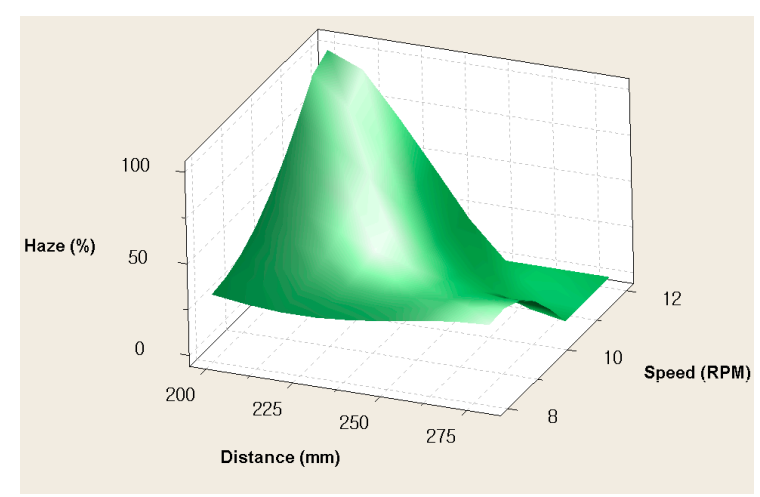

(a)

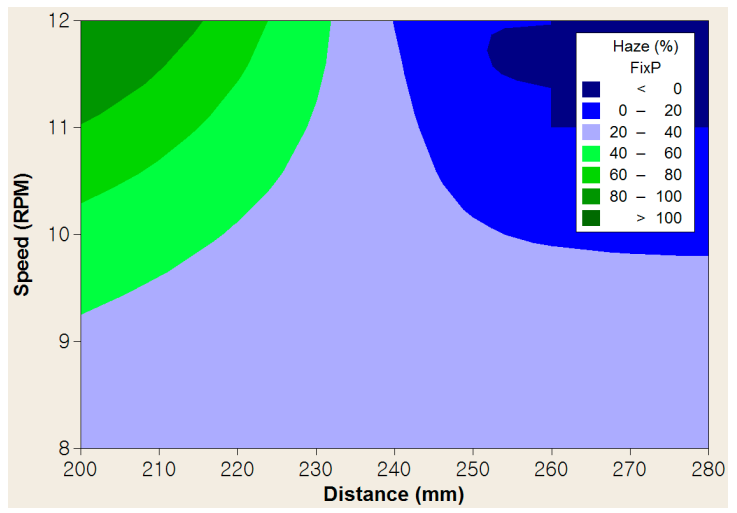

(b)

Figure 5. Effects of the motor speed for control of glass feed speed and the distance of the nozzle from the surface on the haze: (a) 3D surface plot; (b) contour plot.

\subsection{Modeling Performance Test}

Table 6 shows the prediction root mean squared error (RMSE) values of the developed process models, the statistical process model, and the machine learning-based process model. As shown in 
the contour plots of the previous section, the effect on the haze of each process input was similar to both process models, but the level of the sensitivity was different at a specific range. However, as a result of the new experiment for the modeling performance test, the predicted RMSE was within $5 \%$ of both models. When evaluating the modeling performance in terms of prediction RMSE, the statistical process model showed a smaller RMSE in both the process model of \#600 grit size and process model of \#800 grit size.

Table 6. The prediction RMS error values of developed process models.

\begin{tabular}{ccc}
\hline Process to Model & $\begin{array}{c}\text { Prediction RMSE for } \\
\text { Statistical Model }\end{array}$ & $\begin{array}{c}\text { Prediction RMSE for Machine } \\
\text { Learning-Based Model }\end{array}$ \\
\hline$\# 600$ grit size & 2.77 & 4.19 \\
$\# 800$ grit size & 0.90 & 2.97 \\
\hline
\end{tabular}

In general, the statistical RSM only considered the order of the input parameters up to the quadratic level, so in highly non-linear and complex processes, new modeling techniques such as machine learning-based modeling methods are expected to show better prediction results. However, the modeling error between the two models did not make a significant difference, and the prediction error of the statistical model was even smaller. However, there are some additional points to consider for these results to be concluded with this experiment alone. First, while conventional machine learning techniques require a large amount of training data set, the training data set used in this work was very limited to save time and cost for modeling and we used only limited data from the experimental design method and some experimental data from the experience of an expert engineer. In addition, some inputs such as motor speed for the control of the glass feed speed were fixed to constant values based on the decision of the expert engineer, and we could not guarantee that the training data were not uniformly distributed over the entire search space, which may have led to the generalization error in process modeling.

\subsection{Recipe Optimization for Various Haze Targets}

Based on the developed two process models, response surface regression-based statistical model, and machine learning-based process model, the optimal process recipes were defined to satisfy the various haze targets for mass production. In fact, in the case of the sandblasting process considered in this study, since the number of input variables was not large and the value of the input variable had to be selected in the discrete type rather than a continuous real number, we found the optimal process conditions directly using the model's prediction of all possible process variables that can be entered in discrete numbers with the system-defined input resolution. However, at first, we ignored the resolution of input values to see the exact optimal process condition and then used the most similar values to the optimal process condition as the real process inputs.

First, the process conditions that satisfy each haze target were found based on the statistical regression model. Figure 6 is an optimization plot to find the process conditions of the $20 \%$ haze target. The search ranges to find the optimal process conditions were set to $2.5 \sim 4.5 \mathrm{MPa}, 200 \sim 280 \mathrm{~mm}$, and 8.0 12.0 RPM for the pressure of the nozzle, the distance of the nozzle from the surface of the glass, and the motor speed for control of the glass feed speed, respectively. As shown in Figure 6, the process conditions for satisfying the $20 \%$ haze target were $3.5070 \mathrm{MPa}$ of the nozzle pressure, $229.5921 \mathrm{~mm}$ of the distance of the nozzle from the surface of the glass, and 10.0141 of the motor speed for control of the glass feed speed. At this condition, the prediction result of haze was $20.0457 \%$ and the composite desirability value was 0.99543 . This measure of composite desirability (D) is the weighted geometric mean of the individual desirabilities for the responses. Considering the resolution of the available process input values, the actual input process conditions were $3.5 \mathrm{MPa}, 230 \mathrm{~mm}$, and $10 \mathrm{RPM}$ for the pressure of the nozzle, the distance of the nozzle from the surface of the glass, and the motor speed for the control of the glass feed speed, respectively. 


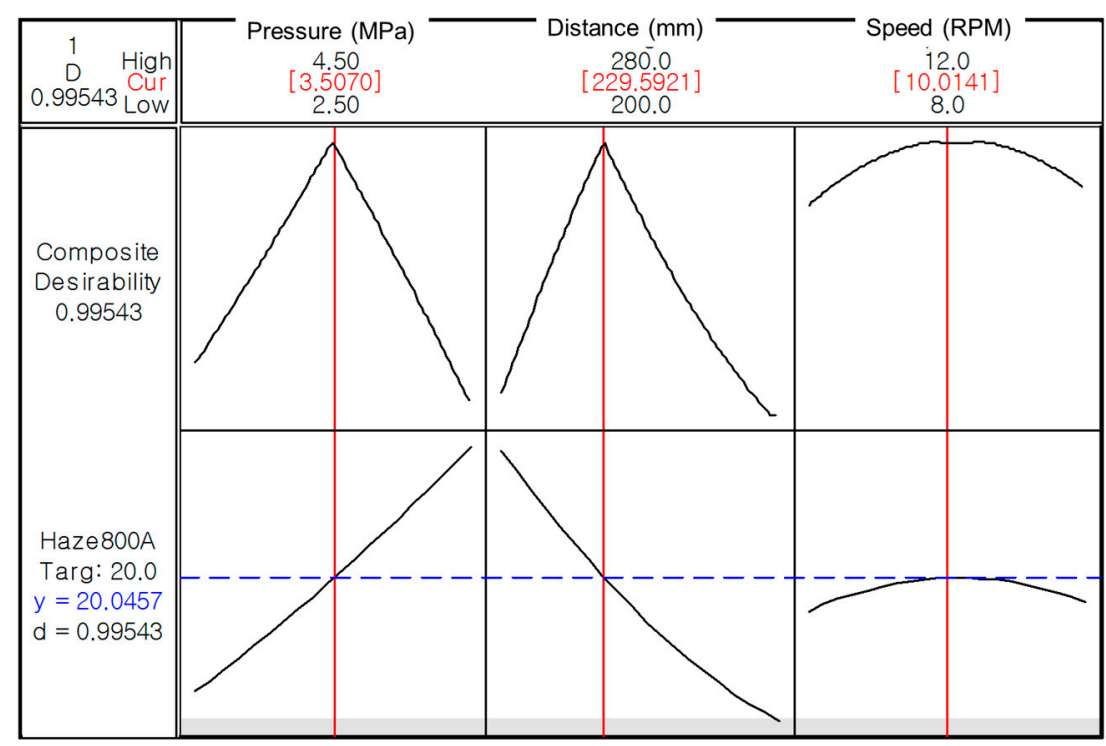

Figure 6. Optimization plot for haze target of $20 \%$.

The process condition found based on the statistical process model was used as an input to the machine learning-based process model, and the deviation between the prediction of two process models was examined. Table 7 shows the haze prediction results of the machine learning-based model for the process input found in the statistical process model. As shown in the table, the prediction results of the machine learning-based model for the $10 \%$ and $20 \%$ haze targets were not significantly different from the process conditions derived from the statistical model, but the difference was about $4.3 \%$ for the $30 \%$ haze target. As noted in the previous section, it can be estimated that the prediction performance of the statistical process model was more reliable because the statistical process model was superior to the prediction RMSE value at the modeling performance test, but the final confirmation was determined through additional experimental results for the review of mass production.

Table 7. Prediction results of the machine running-based process model using process conditions optimized by the statistical process model.

\begin{tabular}{ccccc}
\hline Haze Target & $\begin{array}{c}\text { Nozzle Pressure } \\
\text { (MPa) }\end{array}$ & $\begin{array}{c}\text { Distance of the Nozzle } \\
\text { from the Surface of the } \\
\text { Glass }(\mathbf{m m})\end{array}$ & $\begin{array}{c}\text { Motor Speed for } \\
\text { Control of Glass } \\
\text { Feed Speed (RPM) }\end{array}$ & $\begin{array}{c}\text { Prediction of the Haze from } \\
\text { Machine Learning-Based } \\
\text { Process Model }\end{array}$ \\
\hline $10 \%$ & 2.75 & 250 & 10 & $11.297 \%$ \\
$20 \%$ & 3.5 & 230 & 10 & $20.279 \%$ \\
$30 \%$ & 4.25 & 220 & 10 & $25.678 \%$ \\
\hline
\end{tabular}

\subsection{Performance Test for Mass Production}

Finally, mass production feasibility was evaluated according to the process conditions for each haze target described in the previous section. For mass production feasibility evaluation, five glass samples for each haze target were processed under the same process conditions, and their performance was examined by measurement of haze, uniformity, and transmittance. To evaluate the uniformity of the surface treatment process, five $50 \times 90 \mathrm{~mm}$ glass samples were arranged on a $400 \times 500 \mathrm{~mm}$ panel as shown in Figure 7a, and the haze was measured in three places for each glass as shown in Figure $7 \mathrm{~b}$. 


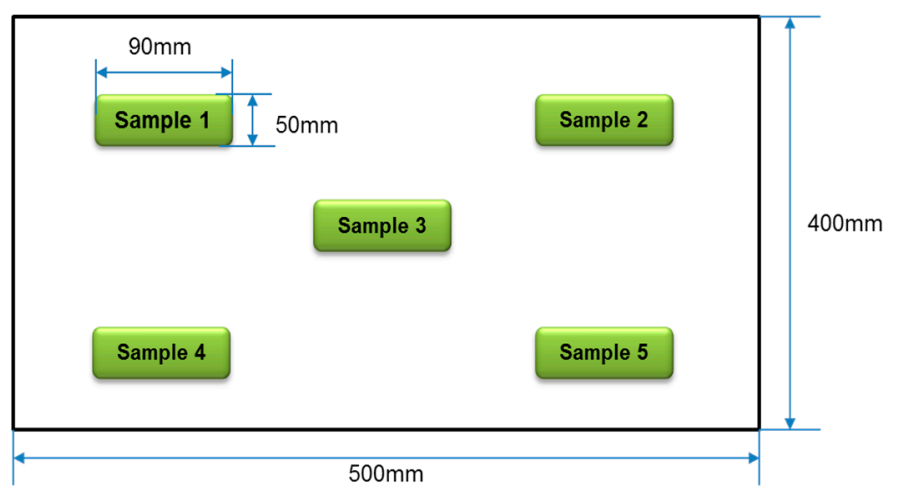

(a)

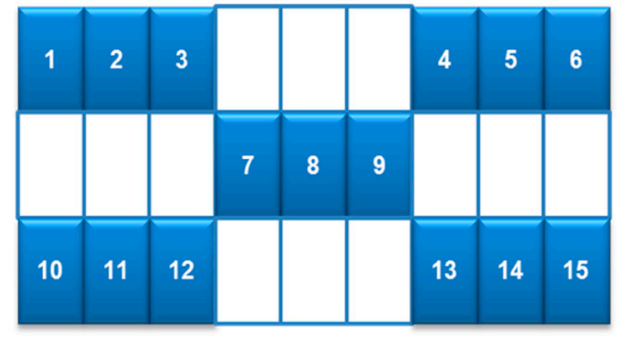

(b)

Figure 7. Layout of uniformity test structure: (a) Dimensions of panel and samples; (b) the measurement location of the haze at each panel for uniformity.

Table 8 shows the results of the sandblasting process for each haze target. The uniformity of the haze was calculated using two methods, Equations (1) and (2).

$$
\begin{gathered}
\text { Uniformity }_{1}(\%)=\left(\frac{H_{\max }-H_{\min }}{2}\right) \times\left(\frac{1}{H_{\text {avg }}}\right) \times 100, \\
\text { Uniformity }_{2}(\%)=\left(\frac{H_{\text {max }}-H_{\text {min }}}{H_{\text {max }}+H_{\text {min }}}\right) \times 100,
\end{gathered}
$$

where $H_{\max }, H_{\min }$, and $H_{a v g}$ represent the maximum value of the haze, minimum value of the haze, and the average value of the haze, respectively.

Table 8. The results of the sandblasting process for haze targets of $10 \%, 20 \%$, and $30 \%$.

\begin{tabular}{ccccccc}
\hline Haze Target (\%) & $\mathbf{H}_{\max } \mathbf{( \% )}$ & $\mathbf{H}_{\min } \mathbf{( \% )}$ & $\mathbf{H}_{\text {avg }}(\mathbf{\%})$ & $\begin{array}{c}\text { Standard } \\
\text { Deviation }\end{array}$ & $\begin{array}{c}\text { Uniformity } \\
\mathbf{( \% )}\end{array}$ & $\begin{array}{c}\text { Uniformity }_{\mathbf{2}} \\
\mathbf{( \% )}\end{array}$ \\
\hline 10 & 11.52 & 10.26 & 10.84 & 0.358 & 5.812 & 5.785 \\
20 & 20.68 & 19.54 & 20.02 & 0.324 & 2.848 & 2.834 \\
30 & 31.54 & 30.14 & 30.86 & 0.461 & 2.268 & 2.270 \\
\hline
\end{tabular}

As shown in the table, the magnitude of the deviation for all targets ranged from $0.14 \%$ to $1.52 \%$, and uniformity did not exceed $6 \%$ for each target, and both the mean and variance were good enough for the mass production of the various target hazes.

Figure 8 shows a comparison between the glass before surface treatment and the glass that was surface treated to have haze values of $10 \%, 20 \%$, and $30 \%$, respectively. When the picture was taken by placing it on the floor, the difference between each glass did not appear on the picture, so the picture was taken after artificially tilting the glass.

Table 9 shows the glossiness and surface roughness of the glass by target haze. For measurement of the glossiness, the gloss meter from Samwon Instruments was used, and for surface roughness expression, arithmetic average roughness ( $\mathrm{Ra}$ ) was calculated with measured values using a laser microscope from Keyence. It can be seen that glossiness decreased rapidly as the haze value increased. Surface roughness was measured through a laser confocal microscope and roughness values (RA) were averaged after measure at the five points per glass. As shown in Table 9, the RA of each glass with three haze targets did not show an intuitive relationship with the haze value and ranged from 0.575 to 0.696. Figure 9 is an example of an RA measurement surface using a laser microscope. Numerically, there was no significant difference in the RA values between glass with a $20 \%$ haze target and glass with a 30\% haze target, but there was some difference in the appearance of the surface observed under a 
microscope. As shown in Figure 9, the surface of Figure 9d showed a higher deviation in the uniformity of surface roughness at some locations compared to that of Figure 9c.

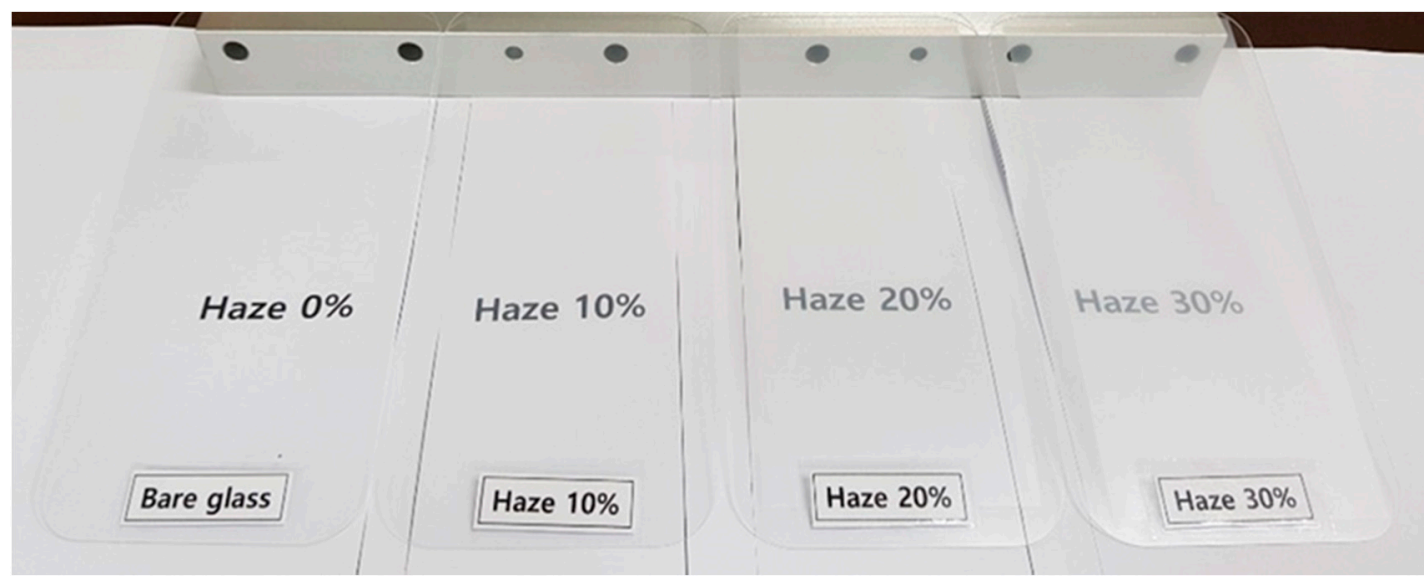

Figure 8. Comparison of surface treatment results according to the haze target value.

Table 9. Glossiness and surface roughness of the glass substrates with various haze targets.

\begin{tabular}{ccc}
\hline Haze Target $(\%)$ & Glossiness $(\mathrm{GU})$ & Surface Roughness $(\boldsymbol{\mu m})$ \\
\hline 0 (Bare glass) & 168.7 & 0.1643 \\
10 & 100.9 & 0.5751 \\
20 & 56.6 & 0.6959 \\
30 & 37.6 & 0.6689 \\
\hline
\end{tabular}

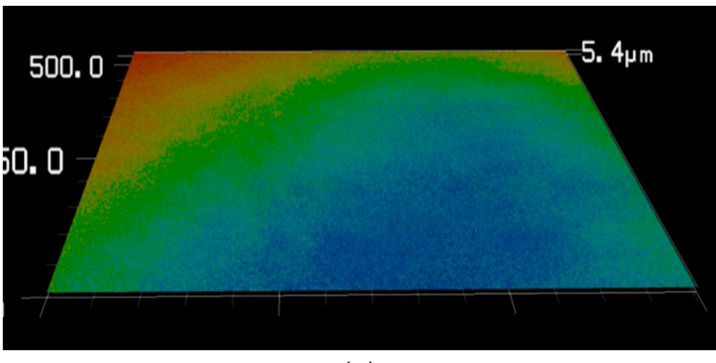

(a)

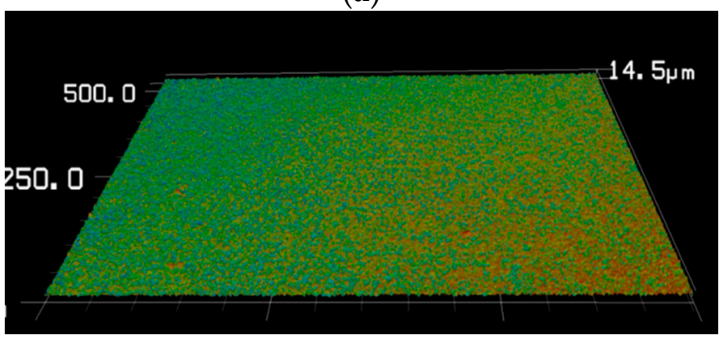

(c)

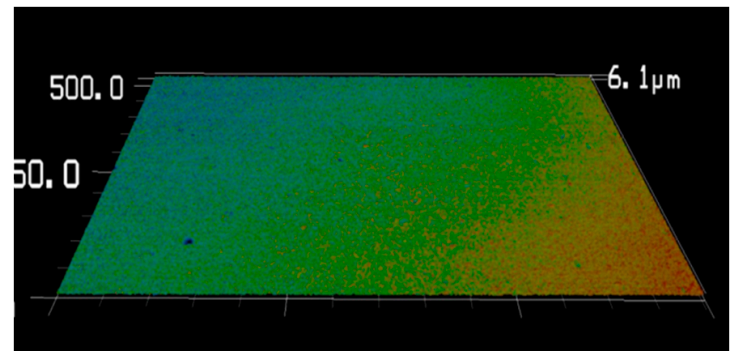

(b)

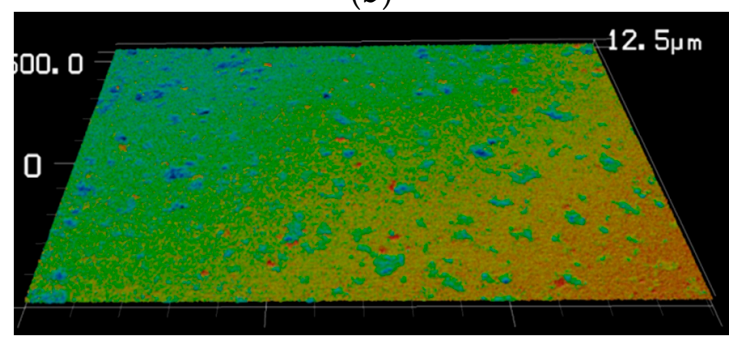

(d)

Figure 9. RA measurement surface: (a) Bare glass; (b) glass with $10 \%$ haze target; (c) glass with $20 \%$ haze target; (d) glass with $30 \%$ haze target.

Figure 10 is the result of the transmittance analysis by wavelength. For measurement of transmittance, a spectrophotometer from Konica Minolta was used and the tested range of wavelengths was from 360 to $740 \mathrm{~nm}$, with a 10-nm wavelength pitch. As shown in this figure, all haze targets showed more than $90 \%$ transmittance within the visible light wavelength band and showed a difference in transmittance of less than 3\% compared to bare glass; thus, the decrease in transmittance by surface treatment for haze was negligible. 


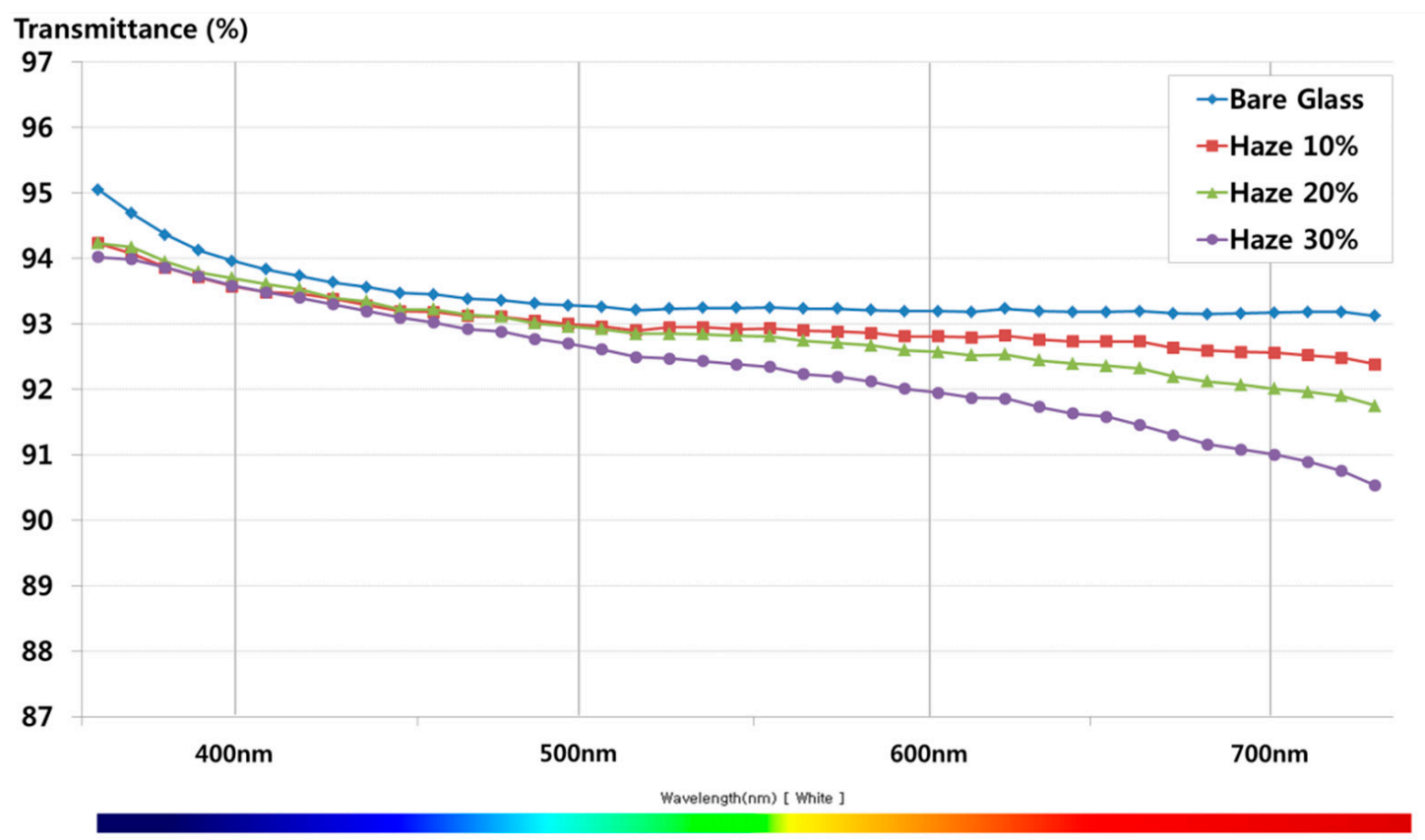

Figure 10. Transmittance analysis plot for various haze targets.

\section{Conclusions}

In this paper, the AG surface treatment technology based on sandblasting was discussed for display devices. By using the physical surface treatment technology instead of the conventional chemical treatment methods, we can apply it on various AG surface treatment applications targeting various haze targets. In order to develop a sandblasting surface treatment technology that satisfies various haze targets, a statistical process model and a machine learning-based process model were developed. Three sets of experiments were conducted for process modeling, the model validation test, and the evaluation of optimized recipes for mass production. The final experiment for mass production showed average haze values of $10.84 \%, 20.02 \%$, and $30.14 \%$, respectively, for the three haze targets of $10 \%, 20 \%$, and $30 \%$, and uniformity was also at least $2.268 \%$ to $5.812 \%$. For the transmittance test, all glasses with different haze targets showed more than $90 \%$ transmittance within the visible light wavelength band and they showed a difference in transmittance of less than $3 \%$ compared to bare glass. Through this work, it is expected that it can be used as a surface treatment technique in various application fields that require various haze values through sandblasting in an environmentally friendly way.

Author Contributions: Conceptualization, C.H.M. and T.S.K.; methodology, C.H.M. and T.S.K.; software, Y.S.K. and T.S.K.; validation, Y.S.K. and C.H.M.; formal analysis, Y.S.K. and C.H.M.; investigation, Y.S.K.; data curation, Y.S.K.; writing — original draft preparation, C.H.M. and T.S.K.; writing—review and editing, T.S.K.; visualization, Y.S.K. and C.H.M.; supervision, C.H.M. and T.S.K.; project administration, C.H.M.; funding acquisition, C.H.M. and T.S.K. All authors have read and agreed to the published version of the manuscript.

Funding: This research was funded by the Catholic University of Korea, Research Fund, 2018, grant number M-2018-B0002-00064. This work was supported by the Ministry of Trade, Industry \& Energy (MI, Korea), the Technology Innovation Program, grant number 10076817.

Conflicts of Interest: The authors declare no conflict of interest. The funders had no role in the design of the study; in the collection, analyses, or interpretation of data; in the writing of the manuscript; or in the decision to publish the results. 


\section{References}

1. Lin, P.; Lin, Y.; Hwang, S.; Jeng, S.; Liao, C. Effects of anti-glare surface treatment, ambient illumination and bending curvature on legibility and visual fatigue of electronic papers. Display 2008, 29, 25-32. [CrossRef]

2. Paz, V.F.; Kohlenbecker, S.; Persidis, E. Implementation of a sparkle characterization method for anti-glare layers on automotive-displays. In Proceedings of the AM-FPD 18, Kyoto, Japan, 3-6 July 2018; pp. 1-4. [CrossRef]

3. Display Quality Assurance Report of American Association of Physicists in Medicine (AAPM). Available online: https://aapm.org/pubs/reports/RPT_270.pdf (accessed on 31 October 2020).

4. Almoallem, Y.D.; Moghimi, M.J.; Jiang, H. Conformal antireflective surface formed onto 3-D silicon structure. J. Microelectromec. Syst. 2018, 27, 380-382. [CrossRef] [PubMed]

5. Isshiki, M. Simulation of anti-glare cover glass using fourier optics consistent with sparkle and other visual performances. Soc. Inf. Disp. Tech. Dig. 2017, 48, 1383-1386. [CrossRef]

6. Raut, H.K.; Ganesh, V.A.; Nair, A.S.; Ramakrishna, S. Anti-reflective coatings: A critical, in-depth review. Energy Environ. Sci. 2011, 4, 3779-3804. [CrossRef]

7. Zhang, X.; Lu, Q.; Cheng, Y.; Shan, Y.; Zhang, G.; Li, D. Moth-eye-like antireflection coatings based on close-packed solid/hollow silica nanospheres. J. Sol-Gel Sci. Technol. 2019, 90, 330-338. [CrossRef]

8. Dobrowolski, J.A.; Poitras, D.; Ma, P.; Vakil, H.; Acree, M. Toward perfect antireflection coatings: Numerical investigation. Appl. Opt. 2002, 41, 3075-3083. [CrossRef]

9. Nuijs, A.M.; Horikx, J.J.L. Diffraction and scattering at antiglare structures for display devices. Appl. Opt. 1994, 33, 4058-4068. [CrossRef]

10. Rudawska, A.; Danczak, I.; Müller, M.; Valasek, P. The effect of sandblasting on surface properties for adhesion. Int. J. Adhes. Adhes. 2016, 70, 176-190. [CrossRef]

11. Dikici, T.; Demirci, S.; Erol, M. Enhanced photocatalytic activity of micro/nano textured $\mathrm{TiO}_{2}$ surfaces prepared by sandblasting/acid-etching/anodizing process. J. Alloys Compd. 2017, 694, 246-252. [CrossRef]

12. Hassan, L.B.; Saadi, N.S.; Karabacak, T. Hierarchically rough superhydrophobic copper sheets fabricated by a sandblasting and hot water treatment process. Int. J. Adv. Manuf. Technol. 2017, 93, 1107-1114. [CrossRef]

13. Cui, Z. Powder Blasting. In Encyclopedia of Microfluidics and Nanofluidics; Li, D., Ed.; Springer: New York, NY, USA, 2015; pp. 2824-2827. [CrossRef]

14. Laouamri, H.; Giljean, S.; Arnold, G.; Kolli, M.; Bouaouadja, N.; Tuilier, M. Roughness influence on the optical properties and scratch behavior of acrylic coating deposited on sandblasted glass. Prog. Org. Coat. 2016, 101, 400-406. [CrossRef]

15. Min, C.; Kim, T. Modeling of sand-blasting process for anti-glare surface treatment of display glass. J. Korean Inst. Surf. Eng. 2018, 51, 303-308. [CrossRef]

16. Whitehouse, D. Surfaces and Their Measurement, 1st ed.; Boston Butterworth-Heinemann: Boston, MA, USA, 2012; pp. 16-24.

17. Sandblasting Grit Conversion Chart. Available online: https://www.raptorblaster.com/sandblasting-gritconversion-chart/ (accessed on 2 November 2020).

18. May, G.S.; Spanos, C.J. Fundamentals of Semiconductor Manufacturing and Process Control, 1st ed.; John Wiley \& Sons: Hoboken, NJ, USA, 2006; pp. 272-331.

19. Hall, M.; Frank, E.; Holmes, G.; Pfahringer, B.; Reutemann, P.; Witten, I.H. The WEKA data mining software. ACM SIGKDD Explor. Newsl. 2009, 11, 10. [CrossRef]

20. Kotthoff, L.; Thornton, C.; Hoos, H.H.; Hutter, F.; Leyton-Brown, K. Auto-WEKA: Automatic Model Selection and Hyperparameter Optimization in WEKA. In Automated Machine Learning. The Springer Series on Challenges in Machine Learning; Hutter, F., Kotthoff, L., Vanschoren, J., Eds.; Springer: Cham, Switzerland, 2019; pp. 81-95. [CrossRef]

21. Gaussian Processes Class. Available online: https://weka.sourceforge.io/doc.dev/weka/classifiers/functions/ GaussianProcesses.html (accessed on 24 November 2020).

22. Kundu, I.; Paul, G.; Banerjee, R. A machine learning approach towards the prediction of protein-ligand binding affinity based on fundamental molecular properties. RRC Adv. 2018, 8, 12127-12137. [CrossRef]

23. Rasmussen, C.E.; Williams, C.K.I. Gaussian Processes for Machine Learning, 1st ed.; The MIT Press: Cambridge, MA, USA, 2016; pp. 33-77. 
24. LWL Class. Available online: https://weka.sourceforge.io/doc.dev/weka/classifiers/lazy/LWL.html (accessed on 24 November 2020).

25. Frank, E.; Hall, M.; Pfahringer, B. Locally weighted naive Bayes. In Proceedings of the Nineteenth Conference on Uncertainty in Artificial Intelligence, Acapulco, Mexico, 7-10 August 2003; pp. 249-256.

Publisher's Note: MDPI stays neutral with regard to jurisdictional claims in published maps and institutional affiliations.

(C) 2020 by the authors. Licensee MDPI, Basel, Switzerland. This article is an open access article distributed under the terms and conditions of the Creative Commons Attribution (CC BY) license (http://creativecommons.org/licenses/by/4.0/). 